\title{
O COMPORTAMENTO ENTRE JUROS E CRÉDITO NO BRASIL PARA CONSUMIDORES E PRODUTORES
}

Karlin Saori Ishii ${ }^{1}$

O mecanismo de crédito viabiliza a atividade econômica moderna através da canalização de recursos para a produção e consumo. O canal de transmissão monetária de juros demonstra que taxas de juros afetam as decisões de tomada de crédito e, consequentemente, afeta a renda. O objetivo deste trabalho foi estudar o relacionamento entre juros e crédito no Brasil de março de 2011 a fevereiro de 2017 através de auto regressão vetorial. Os resultados indicam que os juros influenciam a tomada de crédito, porém o oposto praticamente não se verifica. Ademais, a tomada de crédito do setor produtivo (pessoa jurídica) reage mais a variações de juros que o setor consumidor (pessoa física).

Palavras-chave: Crédito, taxa de juros, VAR

\section{THE BEHAVIOR BETWEEN INTEREST RATE AND CREDIT IN BRAZIL FOR CONSUMERS \\ AND \\ PRODUCERS}

The credit mechanism enables modern economic activity through the channeling of resources into production and consumption. The interest rate channel of monetary transmission mechanism demonstrates that interest rates affect credittaking decisions and, consequently, affect income. The objective of this work was to study the relationship between interest rate and credit in Brazil from March 2011 to February 2017 through vector auto regression. The results indicate that interest rates influence credit, but the opposite is practically non-existent. In addition, credit taking of the productive sector (legal entity) reacts more to interest rate variations than the consumer sector (individual).

Keywords: Credit, interest rate, VAR

E43 Interest Rate: Determination, Term Structure, and Effects

C32 Time-Series Models, Dynamic Quantile Regressions, Dynamic Treatment Effect Models, Diffusion Processes, State Space Models

\section{Introdução}

A estabilidade econômica a partir do Plano Real e, principalmente a estabilidade de preços com o regime de metas de inflação propiciaram um ambiente de estabilidade e controle da taxa de juros a níveis mais baixos que os praticados em períodos anteriores. Apesar de a taxa de juros brasileira ser maior do que a internacional, o ambiente de estabilidade de preços e o aumento da previsibilidade em relação à evolução da economia propiciaram um aumento da contratação de crédito que elevou os níveis de investimento.

\footnotetext{
${ }^{1}$ Doutora em Economia pela Escola Superior de Agricultura Luiz de Queiroz/Universidade de São Paulo. Professora da Universidade Federal de Mato Grosso. Email: ishiisaori@hotmail.com
}

Revista de Estudos Sociais | Ano 2017, N. 39, V. 20, Pag. 165 
Além do aumento dos níveis de investimento, o ambiente de estabilidade econômica gerou um aumento de consumo, pois a estabilidade de preços foi responsável pelo aumento da contratação de crédito de pessoa física, pois possibilitou-se o alongamento do horizonte de planejamento de gastos.

Figura 1 - Evolução do crédito no Brasil

\section{EVOLUÇAO DA COMPOSIÇAO DO CREDITO}

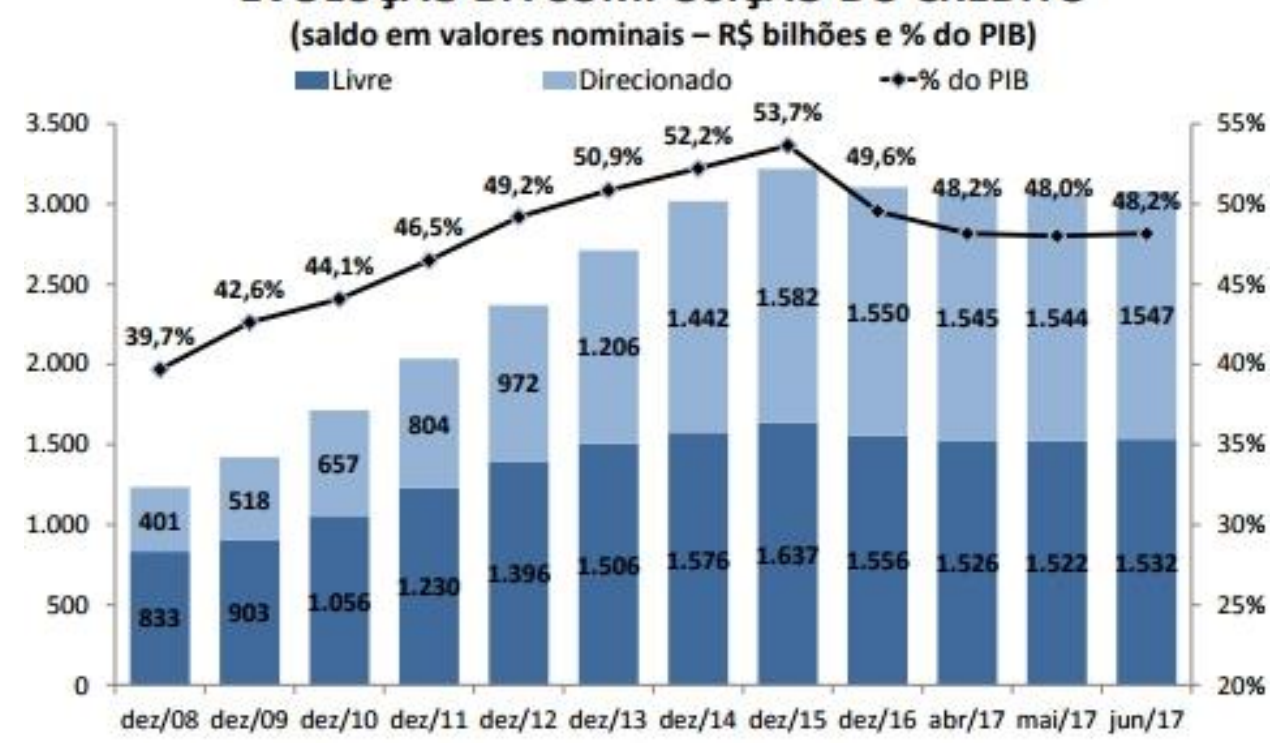

Fonte: Secretaria de Política Econômica - Ministério da Fazenda

Observando a Figura 1, é possível perceber o crescimento do crédito que passa dos 39,7\% do PIB em 2008 para 53,7\% do PIB em dezembro de 2015. No entanto, verificou-se que a partir de 2016, devido à crise, o crédito contratado no país sofre uma redução. Além disto, destaca-se o alto crescimento do montante de recursos direcionados de crédito (habitação, rural e BNDES) entre 2008 a 2015.

Neste sentido é crucial o papel do sistema de intermediação dos recursos financeiros entre os agentes econômicos superavitários e deficitários. Sendo assim, o mercado financeiro permite que um agente econômico (consumidor ou firma) que não tenha possibilidade de aplicar recursos próprios em algum empreendimento seja colocado em contato com um agente superavitário disposto a fornecer recursos mediante uma compensação financeira (juros).

Segundo os dados do Banco Central, as operações de crédito do sistema financeiro nacional totalizaram $43,7 \%$ do PIB em dezembro de 2009, chegando a $54,5 \%$ do PIB em dezembro de 2015. Em 2015, a média de recursos de crédito do sistema financeiro nacional para pessoa jurídica foi de $28,3 \%$ do PIB enquanto que para pessoa jurídica foi de $25,2 \%$. Sendo assim, ambos os tipos de crédito tiveram participação significativa no PIB.

A contratação de crédito é feita mediante o pagamento de juros. Sendo assim, a taxa de juros tem um papel fundamental para esta intermediação. $O$ principal mecanismo de transmissão da política monetária, que é o canal de juros, mostra que variações na taxa de juros causam variações na contratação de crédito que por sua vez causam variações nos investimentos e no produto [Mishkin (1998), Carvalho et al (2007)], ou ainda, uma elevação na taxa de juros reduz a contratação de crédito, o investimento/consumo e, portanto, o produto.

Revista de Estudos Sociais | Ano 2017, N. 39, V. 20, Pag. 166 
Sendo assim, o conhecimento da magnitude do impacto dos juros sobre a tomada de crédito é importante para conhecer os possíveis efeitos sobre a renda.

A motivação de produtores e consumidores sobre a tomada de crédito é distinta, sendo que os produtores utilizam o crédito para viabilizar a produção enquanto que os consumidores tomam crédito para aumentar a satisfação. Portanto, a análise foi realizada para ambos os tomadores de crédito separadamente.

Sendo assim, este trabalho busca verificar o relacionamento entre taxa de juros e crédito no Brasil 2011 a 2017 para pessoa física (PF) e pessoa jurídica (PJ).

\section{Mecanismo de transmissão da política monetária}

Nos anos 1950 foi desenvolvida a teoria monetarista segundo a qual a política monetária deveria ser utilizada para a estabilidade de preços. Neste sentido, o ideal é que se estabeleça regras de política monetária. Sendo assim, não deveriam ser realizadas políticas discricionárias que poderiam ser desestabilizadoras e inflacionárias.

Verifica-se que na atualidade, poucos autores utilizam a teoria monetarista como embasamento para trabalhos na área econômica. O corpo teórico monetarista foi gradativamente substituído pelo novo-clássico e novokeynesiano que apresentavam pressupostos mais realistas para a explicação do comportamento econômico.

Porém, os monetaristas foram os responsáveis pelo acirramento das discussões sobre a eficácia ou ineficácia da política monetária com estudos que buscavam "desenvolver melhor compreensão dos canais (além dos efeitos das taxas de juros sobre investimentos) através das quais a política monetária influencia a demanda agregada". (Mishkin, 1998, p. 386)

Para esta escola, o papel da política monetária era de grande relevância para a coordenação macroeconômica. Ao contrário dos keynesianos que acreditavam que a política fiscal era o instrumento chave para a condução macroeconômica, Friedman acreditava no poder da política monetária na gestão da economia. Para este autor, o multiplicador bancário deveria inclusive ser abolido ou controlado com rigor para que o controle monetário fosse mais efetivo. Segundo Cutruneo (2010):

\footnotetext{
"ao abolir o multiplicador os BCs teriam ainda mais poder sobre a política monetária, o que de um lado seria positivo, dado que traria mais capacidade à autoridade monetária em termos de meios de se alcançar a estabilidade, mas por outro lado seria negativo, já que um BC com grande poder em mãos pode gerar tanto estabilização quanto grande instabilidade" (Cutruneo, 2010, p.16)
}

A proposta de Friedman (1960) de controle monetário não era relacionada com a utilização ativa da política monetária, mas com a proposta de utilização de regras de controle do crescimento do estoque monetário como forma de evitar a discricionariedade e, portanto a desestabilização. Ademais, a utilização de regras, para Friedman, é uma forma de evitar conceder um poder excessivo aos bancos centrais e, por conseguinte, levar um grupo pequeno de pessoas a agir segundo os próprios interesses. Com isso, seria garantida uma maior liberdade

Revista de Estudos Sociais | Ano 2017, N. 39, V. 20, Pag. 167 
econômica a individual, como é o caso da melhoria no planejamento econômico individual.

Porém, uma das dificuldades de sustentação da teoria monetarista é o crescimento do mercado financeiro na década de 1980. Com o mercado financeiro aumentando, a demanda de moeda torna-se instável e isto faz com que as regras monetárias sejam incompatíveis com o objetivo de estabilidade econômica.

Outra mudança no ambiente econômico é relacionada com a expectativa. Monetaristas trabalham com expectativas adaptativas em que o agente observa o que vem ocorrendo para determinar a sua ação futura. Em um ambiente de instabilidade de demanda de moeda, a formação de expectativas adaptativas leva o agente a cometer erros sistemáticos, e, portanto, desestabilizadores, $\mathrm{O}$ fato de a economia estar mais instável do que nos anos 1950-1960, faz com que a teoria monetária não seja eficaz.

Lucas (1972), adepto da teoria novo-clássica, enfatiza que os agentes formam as expectativas baseadas em toda informação disponível e, que acertam em média. Isto significa que o trade off entre inflação e desemprego de curto prazo não se verifica. Portanto, para esta escola de pensamento, as políticas econômicas discricionárias não são praticáveis. Este fenômeno é conhecido como postulado da ineficácia das políticas econômicas. Sendo assim, novoclássico refuta de forma mais contundente a utilização da política monetária de forma ativa.

A escola novo-keynesiana que surge logo a seguir critica o postulado de ineficácia das políticas econômicas. Para os novos keynesianos, a demanda agregada é instável e há rigidezes de preços e salários que não permitem os ajustamentos rápidos de curto prazo. Portanto, os desajustamentos na economia precisam ser controlados com política econômica ativa, visto que demoram a desaparecer.

Segundo Wooford (2003), o controle da moeda via regras de emissão monetárias não é a ideal devido à instabilidade da demanda de moeda. Apesar disto, os novo-keynesianos concordam com a adoção de regra de política monetária e o controle dos bancos centrais relativos à estabilidade de preços. Duas regras que podem ser utilizadas são a meta de inflação que é o anúncio público de meta de taxa de inflação e a regra de Taylor que é a utilização da taxa de juros como forma de estabilização de preços. Sendo assim, na maior parte do tempo a taxa de juros é utilizada como instrumento de política monetária e, portanto comporta-se como uma variável exógena.

A visão tradicional de transmissão monetária keynesiana é tal que uma expansão monetária( $\uparrow \mathrm{M})$ faz com que haja queda nas taxas de juros nominais $(\downarrow$ i). No curto prazo existe rigidez de preços, de forma que há uma queda nas taxas reais de juros $(\downarrow$ r), que geram redução no custo de captação do capital, gerando aumento nos níveis de ( $\uparrow$ I)investimento, levando ao aumento da demanda agregada e do produto( $\uparrow$ Y). Este é o canal de transmissão de juros.

$$
\uparrow M \rightarrow \downarrow i \quad \cdots \downarrow r \rightarrow \uparrow I \quad \cdots \uparrow Y
$$

Segundo Mishkin (1998), originalmente Keynes utilizava o mecanismo para analisar questões empresariais, porém este mesmo mecanismo de

Revista de Estudos Sociais | Ano 2017, N. 39, V. 20, Pag. 168 
transmissão pode ser utilizado também para as decisões dos consumidores em relação a gastos com moradia e bens duráveis, de forma que I pode ser considerado como gastos com bens imóveis e com bens duráveis.

\section{Revisão de literatura}

Há diversos trabalhos econômicos relacionados ao crédito, com enfoques no setor rural, no setor bancário e relacionados ao consumidor. Será realizado aqui uma breve revisão sobre estes estudos.

Gonçalves Junior et al (2008) estudaram as características dos tomadores de empréstimos rurais e a possibilidade de previsão da inadimplência para uma cooperativa do Paraná, utilizando a técnica de regressão logística, verificando que os produtores de laranja foram os mais suscetíveis a inadimplência. Há ainda Junqueira e Abramovay (2005) que analisam a sustentabilidade do sistema Cresol de cooperativa de crédito rural.

Sbicca, Floriani e Juk (2012) avaliam a vulnerabilidade do consumidor no ambiente de extensa expansão de crédito ocorrido no Brasil nos anos 2000 verificando a vulnerabilidade dos agentes de baixa renda.

Ferreira et al (2011) avaliam o risco de crédito observando a inadimplência, para tanto buscaram definir o perfil de clientes com risco de inadimplência futura para empréstimos em um banco de varejo de forma a auxiliar na decisão de concessão de crédito.

Bischoff (2015) estudou o risco bancário e a taxa de juros observando que a redução da taxa de juros acarretou em incentivos para as instituições financeiras assumirem maiores riscos de 2003 a 2013.

Monticelli (2003) estudou o relacionamento entre crédito, juros e inadimplência observando que no mercado de crédito ocorre um circulo vicioso em que a inadimplência leva os agentes financeiros a cobrarem spread maior que por sua vez acarretam o aumento da taxa de juros cobrado e que levam ao aumento posterior da inadimplência.

Nascimento (2015) estudou a evolução do crédito no Brasil mostrando o comportamento do spread bancário e a atuação do governo brasileiro no combate à crise financeira internacional. Tavares, Montes e Guillen (2013) também analisam o spread indicando que a política monetária afeta o prêmio de seguro de crédito, existindo uma relação positiva entre o prêmio e o spread.

Pereira e Wagner (2012) estudaram a relação de causalidade entre a disponibilidade de crédito e a inadimplência a fim de verificar o racionamento de crédito no Brasil. Utilizaram um vetor auto regressivo para verificar a persistência do impacto da inadimplência sobre o crédito. Tabak, Craveiro e Cajueiro (2010) procuraram estimar a relação entre créditos inadimplentes e eficiência bancária, avaliando a eficiência bancária através de um modelo Análise Envoltória de Dados (DEA)

Santanna, Borça junior e Araujo (2009) estudaram o mercado de crédito no Brasil enfatizando o papel do BNDES entre 2004 e 2008.

Há trabalhos que estudam a transmissão monetária como Tomazzia e Meurer (2009) que verificam como o setor industrial reage a mudanças de juros utilizando o VAR que é a mesma metodologia utilizada neste trabalho.

\section{Metodologia e dados}

Revista de Estudos Sociais | Ano 2017, N. 39, V. 20, Pag. 169 
Os dados mensais utilizados no trabalho são de concessão de crédito e taxa de juros totais para o Brasil e para pessoa física e pessoa jurídica também a nível nacional disponibilizados pelo Banco Central do Brasil para o período de março de 2011 a fevereiro de 2017.

Segundo o Banco Central do Brasil:

"o arcabouço de definições de caráter metodológico bem como as instruções para o fornecimento do novo conjunto de dados por parte das instituições financeiras foi publicado entre dezembro de 2008 e outubro de 2009. O envio das novas informações teve início em março de 2010, em caráter preliminar, e o processo de validação prosseguiu até o final de 2012". (BACEN, 201-)

Sendo assim, não foi possível utilizar dados anteriores a este período, devido à mudança na metodologia.

Para a análise econométrica foi utilizado o VAR bivariado buscando compreender o relacionamento entre crédito e taxa de juros. Foram estimados 3 autorregressões vetoriais para os seguintes relacionamentos: juros total e crédito total; juros para pessoa física e crédito para pessoa física e; juros para pessoa jurídica e crédito para pessoa jurídica.

A metodologia foi retirada de Ishii (2008) e foi baseada em Enders (2004). Para que se possa utilizar o modelo VAR, é necessário que as séries utilizadas sejam estacionárias, para que não haja o problema de regressão espúria. Portanto, a verificação da estacionariedade foi feita através do Teste Dickey Fuller Aumentado. Segundo Enders (2004), existem vários métodos para a determinação da defasagem a ser utilizada no Teste Dickey Fuller Aumentado como o Akaike Information Criterion - AIC, Schwartz Bayesian Criterion - SBC, Lagrange Multiplier -LM entre outros.

A partir daí, realiza-se o teste Dickey Fuller através das seguintes equações:

$$
\begin{aligned}
& \Delta y_{t}=\gamma y_{t-1}+\sum_{i=2}^{p} \beta_{i} \Delta y_{t-i+1}+\varepsilon_{t} \\
& \Delta y_{t}=a_{0}+\gamma y_{t-1}+\sum_{i=2}^{p} \beta_{i} \Delta y_{t-i+1}+\varepsilon_{t} \\
& \Delta y_{t}=a_{0}+\gamma y_{t-1}+a_{2} t+\sum_{i=2}^{p} \beta_{i} \Delta y_{t-i+1}+\varepsilon_{t}
\end{aligned}
$$

As equações (1) a (3) são utilizadas para verificar a existência de raiz unitária. A primeira equação é um passeio aleatório puro, a segunda tem um termo de intercepto $a_{0}$ e a terceira possui um termo de intercepto $a_{0}$ e um termo de tendência linear $a_{2} t$. Para verificar se há raiz unitária nas séries, a hipótese é de que $\gamma=0$ e utiliza-se a estatística $\tau$ para $a_{0}=a_{2}=0$, a estatística $\tau_{\mu}$ para $a_{2}=0$ e $\tau_{\tau}$ para a equação completa. Se for indicada a presença de raiz unitária, realizam-se novamente os testes com mais uma diferença nas séries. Se após essa diferença, a série tornar-se estacionária, diz-se que a série é uma I(1), ou série integrada de ordem um.

O processo gerador das séries de tempo é estacionário se suas características não se alteram com o tempo. Assim, um processo $y_{t}$ será estacionário se possuir média e variância constantes ao longo do tempo e a Revista de Estudos Sociais | Ano 2017, N. 39, V. 20, Pag. 170 
covariância entre os valores da série depender apenas da distância de tempo que separa os dois valores e não dos tempos reais em que as variáveis são observadas, de forma que:

$$
\begin{array}{lr}
E\left(y_{t}\right)=\mu & \text { Média Constante } \\
\operatorname{Var}\left(y_{t}\right)=\sigma^{2} & \text { Variância constante } \\
\operatorname{Cov}\left(y_{t}, y_{t+s}\right)=\operatorname{Cov}\left(y_{t+j}, y_{t+j-s}\right) & \text { Covariância depende de s e não de j }
\end{array}
$$

O teste de hipótese realizado para examinar a estacionariedade da série foi baseado nas distribuições que constam em Dickey e Fuller $(1979,1981)$ e Fuller (1976). O teste de raiz unitária tem por objetivo averiguar a estacionariedade das séries temporais. Caso a variável não seja estacionária, de forma geral, diferenciando-a certo número de vezes, ela tornar-se-á estacionária. O número de vezes que a série deve ser diferenciada é a ordem de integração dessa variável.

Se as variáveis são integradas (estacionárias após uma certa quantidade de diferenças) de mesma ordem, pode-se testar a existência de co-integração entre elas.

Para atender aos objetivos do trabalho, como dito anteriormente, utilizou a Auto-regressão Vetorial - VAR. Um modelo VAR usual descreve os movimentos dinâmicos das variáveis endógenas através de seus próprios valores passados. Utilizando Enders (2004), expressa-se um sistema bivariado simples:

$$
\begin{aligned}
& y_{t}=b_{10}+b_{12} z_{t}+\gamma_{11} y_{t-1}+\gamma_{12} z_{t-1}+\varepsilon_{y t} \\
& z_{t}=b_{20}+b_{21} y_{t}+\gamma_{21} y_{t-1}+\gamma_{22} z_{t-1}+\varepsilon_{z t}
\end{aligned}
$$

Assume-se que $y_{t}$ e $z_{t}$ são estacionários; $\varepsilon_{y t}$ e $\varepsilon_{z t}$ são distúrbios ruído branco com erro-padrão de $\sigma_{y}$ e $\sigma_{z}$; e que a seqüência $\left\{\varepsilon_{y t}\right\}$ e $\left\{\varepsilon_{z t}\right\}$ são distúrbios não-correlacionados do tipo ruído branco.

Porém, as equações (7) e (8) são a forma estrutural ou modelo primitivo do VAR, em que $y_{t}$ tem efeito contemporâneo em $z_{t}$ e $z_{t}$ tem efeito contemporâneo em $y_{t}$. Para calcular o modelo é necessário transformá-lo de forma a obter um VAR na forma padrão:

$$
x_{t}=A_{0}+A_{1} x_{t-1}+e_{t}
$$

que na forma de equações seria:

$$
\begin{aligned}
& y_{t}=a_{10}+a_{11} y_{t-1}+a_{12} z_{t-1}+e_{1 t} \\
& z_{t}=a_{20}+a_{21} y_{t-1}+a_{22} z_{t-1}+e_{2 t}
\end{aligned}
$$

Porém, não é possível identificar todos os parâmetros do modelo primitivo através da estimação do VAR padrão devido ao problema de subidentificação.

Revista de Estudos Sociais | Ano 2017, N. 39, V. 20, Pag. 171 
Um meio de identificar o modelo é utilizar um sistema recursivo proposto por Sims (1980), através de restrições na matriz de relações contemporâneas.

Com a imposição das restrições, a decomposição dos resíduos torna-se uma matriz triangular conhecida como Decomposição de Choleski. Há outros formas de solucionar o problema da identificação do VAR, porém como será estimado um VAR bivariado, esse sistema recursivo é viável.

Como são necessários a decomposição da variância e o impulso resposta para atender os objetivos do trabalho, é imprescindível a imposição das restrições sobre a matriz de relações contemporâneas de modo a tornar 0 sistema primitivo identificado.

A função impulso-resposta do modelo VAR é escrita a partir de uma representação média-móvel em que as variáveis $y_{t}$ e $z_{t}$ são expressas em termos dos valores correntes e passados de $e_{1 t}$ e $e_{2 t}$ Pode-se representa-la por:

$$
\left[\begin{array}{l}
y_{t} \\
z_{t}
\end{array}\right]=\left[\begin{array}{l}
\bar{y} \\
\bar{z}
\end{array}\right]+\sum_{i=0}^{\infty}\left[\begin{array}{ll}
\phi_{11}(i) & \phi_{12}(i) \\
\phi_{21}(i) & \phi_{22}(i)
\end{array}\right]^{i}\left[\begin{array}{c}
\varepsilon_{y t-i} \\
\varepsilon_{s t-i}
\end{array}\right]
$$

ou na forma compacta:

$$
x_{t}=\mu+\sum_{i=0}^{\infty} \phi_{i} \varepsilon_{t-i}
$$

Os coeficientes de $\phi_{i}$ podem ser utilizados para gerar os impactos de $\varepsilon_{y t}$ e $\varepsilon_{z t}$ sobre as seqüências $\left\{y_{t}\right\}$ e $\left\{z_{t}\right\}$. São, portanto, conhecidos como multiplicadores de impacto.

Os erros de previsão são importantes para descobrir as inter-relações entre as variáveis no sistema. Para a seqüência $\left\{y_{t}\right\}$ pode-se verificar que o erro de previsão $n$ períodos a frente é dado por:

$$
\begin{aligned}
& y_{t+n}-E_{t} y_{t+n}=\phi_{11}(0) \varepsilon_{y t+n}+\phi_{11}(1) \varepsilon_{y t+n-1}+\ldots+\phi_{11}(n-1) \varepsilon_{y t+1}+ \\
& +\phi_{12}(0) \varepsilon_{z t+n}+\phi_{12}(1) \varepsilon_{z t+n-1}+\ldots+\phi_{12}(n-1) \varepsilon_{z t+1}
\end{aligned}
$$

E a variância do erro de previsão de $y_{t+n}$ para $n$ períodos a frente é:

$$
\begin{aligned}
& \sigma_{y}(n)^{2}=\sigma_{y}^{2}\left[\phi_{11}(0)^{2}+\phi_{11}(1)^{2}+\ldots+\phi_{11}(n-1)^{2}\right]+ \\
& +\sigma_{z}^{2}\left[\phi_{12}(0)^{2}+\phi_{12}(1)^{2}+\ldots+\phi_{12}(n-1)^{2}\right]
\end{aligned}
$$

Pelo fato de todos os valores serem não negativos, a variância do erro de previsão é crescente em relação à $n$. Além disso, é possível separar a variância do erro de previsão em proporção ao choque $\varepsilon_{y t}$ e $\varepsilon_{z t}$, respectivamente.

$$
\frac{\sigma_{y}^{2}\left[\phi_{11}(0)^{2}+\phi_{11}(1)^{2}+\ldots+\phi_{11}(n-1)^{2}\right]}{\sigma_{y}(n)^{2}}
$$

Revista de Estudos Sociais | Ano 2017, N. 39, V. 20, Pag. 172 


$$
\frac{\sigma_{z}^{2}\left[\phi_{12}(0)^{2}+\phi_{12}(1)^{2}+\ldots+\phi_{12}(n-1)^{2}\right]}{\sigma_{y}(n)^{2}}
$$

Sendo assim, é possível conhecer a proporção dos movimentos na série $\left\{y_{t}\right\}$ devido ao seu próprio choque $\varepsilon_{y t}$ ou ao choque na outra variável $\varepsilon_{z t}$. E ainda, se o choque em $\varepsilon_{z t}$ não explicar em nada a variância do erro de previsão para a seqüência $\left\{y_{t}\right\}$ em qualquer que seja o horizonte de previsão, diz-se que a seqüência $\left\{y_{t}\right\}$ é exógena, ou dito de outra forma, $\left\{y_{t}\right\}$ evolui independentemente dos choques $\varepsilon_{z t}$ e da seqüência $\left\{z_{t}\right\}$.

Se uma série é não estacionária, a tendência estocástica pode ser eliminada através da diferenciação. Para os casos de estimação de VAR contendo variáveis não estacionárias, é possível que haja combinações lineares estacionárias para variáveis integradas de mesma ordem, ou seja, relações de equilíbrio de longo prazo que devem ser incluídas no modelo para evitar erros de especificação.

Um dos métodos mais utilizados para verificar a cointegração é o de Johansen (1988). Este método consiste na utilização de estimadores de máxima verossimilhança para testar a presença de cointegração e estimar vetores.

\section{A economia brasileira pós Plano Real}

O período de implantação do Plano Real foi conturbado no que se refere ao fornecimento de crédito. Segundo Cysne e Costa (1997), o plano real com o compromisso de estabilização imediata de preços foi amparado na pressuposição de que houvessem medidas posteriores na área fiscal. Porém, o atraso da reforma no sistema fiscal trouxe custos para o combate à inflação que afetaram com intensidade o sistema financeiro.

Em relação ao comportamento do consumidor, 1995 foi um ano de grande instabilidade creditícia. Houve uma onda consumista baseada em crédito fácil. Segundo Cysne e Costa (1997, p.325) "os consumidores pareciam ter esquecido que embora o valor das prestações fosse fixo, os rendimentos nominais não mais aumentariam como antes". Além disso, houve um crescimento residual dos preços principalmente dos serviços, ocorrendo uma perda do salário real e uma redução da capacidade do salário de saldar dívidas. Com isso, houve a elevação da inadimplência.

Ainda segundo Cysne e Costa (1997), os consumidores se sentiram enriquecidos com a queda do imposto inflacionário e com a valorização cambial, gerando um período de euforia juntamente com um período de inadimplência.

Quando se observa a concessão de crédito, o Plano Real trouxe um grande impacto sobre o funcionamento bancário, segundo Corazza (2000). Os bancos precisaram adaptar-se à queda abrupta da receita inflacionária, readaptar sua estrutura, reduzir custos e procurar novas fontes de receitas. Segundo Corazza (2000, p.4):

Revista de Estudos Sociais | Ano 2017, N. 39, V. 20, Pag. 173 
"em termos do valor da produção bancária imputada, as receitas inflacionárias, que representavam $87,3 \%$, reduziram-se para $49,5 \%$ em 1994 e 1,6\% em 1995".

Neste processo, houve a extinção de diversas instituições financeiras. Ademais, houve a abertura ao capital externo e o ingresso de bancos estrangeiros que aumentaram a competição, levando as instituições menos eficientes a extinção.

Segundo Corazza (2000, p.2), "as crises bancárias afetam a economia de forma mais profunda que as crises de outras empresas, pois afetam o crédito e os juros, os investimentos e o nível de atividade".

A política monetária deveria ser utilizada como instrumento para estabilidade de preços. Neste sentido, a política de metas de inflação é uma declaração de que a economia prioritariamente busca a estabilidade de preços. Se há a credibilidade na condução desta política monetária, cria-se um ambiente propício para o investimento, que pode gerar um aumento da tomada de crédito pelos investidores. Ademais, um ambiente de estabilidade de preços gera um aumento da tomada de créditos também pela pessoa física.

\section{Resultados}

O primeiro teste econométrico necessário antes da estimação dos modelos VAR/VEC é o teste de Dickey Fuller Aumentado. Este é um teste de verificação de estacionariedade das séries.

Tabela 1- Teste de Dickey Fuller Aumentado com dummies de novembro a fevereiro

\begin{tabular}{|c|c|c|c|}
\hline & Lags & Séries em nível & $\begin{array}{l}\text { Séries em primeira } \\
\text { diferença }\end{array}$ \\
\hline & LM & $\tau_{\tau}$ & $\tau$ \\
\hline Crédito total & 6 & -0.57071 & -4.83056 \\
\hline $\begin{array}{l}\text { Crédito } \\
\text { pessoa } \\
\text { juridica }\end{array}$ & 8 & -0.60698 & -3.05045 \\
\hline $\begin{array}{l}\text { Crédito } \\
\text { pessoa física }\end{array}$ & 1 & -2.23501 & -12.52737 \\
\hline Juros total & 4 & -2.27737 & -2.28001 \\
\hline $\begin{array}{l}\text { Juros pessoa } \\
\text { jurídica* }\end{array}$ & $0^{*}$ & -1.63564 & -7.81530 \\
\hline $\begin{array}{l}\text { Juros pessoa } \\
\text { fisica }\end{array}$ & & -1.68903 & -4.81412 \\
\hline
\end{tabular}

Fonte: Elaboração própria

Nota: Valores críticos $\tau_{\tau}=-3,45$ e $\tau=-1,95$. * Foi utilizado o Bayesian Information criterion, pois os resultados obtidos pelo critério Lagranger multiplier faziam com que houvesse problemas de estabilidade do VAR.

$\mathrm{Na}$ Tabela 1 constam os resultados do teste de Dickey Fuller Aumentado para as séries que serão utilizadas nos modelos VAR. Os testes para a definição de defasagens indicam que há a necessidade de incluir de 2 a 8 lags nas Revista de Estudos Sociais | Ano 2017, N. 39, V. 20, Pag. 174 
equações de teste pelo critério do multiplicador de Lagrange. Porém, para juros de pessoa jurídica foi utilizado o critério de informação bayesiano e isso ocorreu pela falta de estabilidade do VAR quando utilizado o critério de Lagrange. Pelos testes Dickey e Fuller Aumentado é possível verificar que todas as variáveis possuem raiz unitária em nível. Porém, todas as variáveis tornam-se estacionárias quando diferenciadas.

\section{Crédito total}

Uma vez que as séries utilizadas são I(1), o próximo passo é verificar se há um vetor de cointegração a ser incluído no modelo, de forma que seja estimado um VAR ou um VEC.

Tabela 2 - Teste de Cointegração

\begin{tabular}{l|l|l|l|l|l|l|l}
\hline p-r & r & Autovalor & Traço & Traço* $^{*}$ & Perc95 & P-Valor & P-Valor \\
\hline 2 & 0 & 0.210 & 19.481 & 17.407 & 20.164 & 0.063 & 0.119 \\
\hline 1 & 1 & 0.046 & 3.251 & 2.730 & 9.142 & 0.545 & 0.638 \\
\hline
\end{tabular}

Fonte: Elaboração própria

Não foi possível verificar cointegração entre as variáveis crédito total de juros total, pois o p-valor indica a não-rejeição da hipótese nula de $r=0$, portanto foi estimado a seguir um modelo VAR em diferença.

Tabela 3 - Decomposição da variância da série juros total

\begin{tabular}{l|l|l|l}
\hline Passos & Erro padrão & Juros total & Crédito total \\
\hline 1 & 0,496191 & 100 & 0 \\
\hline 2 & 0,533395 & 90,577 & 9,423 \\
\hline 3 & 0,534039 & 90,572 & 9,428 \\
\hline 4 & 0,559524 & 91,282 & 8,718 \\
\hline 5 & 0,562363 & 90,698 & 9,302 \\
\hline 6 & 0,562935 & 90,716 & 9,284 \\
\hline 7 & 0,565411 & 90,764 & 9,236 \\
\hline 8 & 0,565721 & 90,715 & 9,285 \\
\hline 9 & 0,565866 & 90,719 & 9,281 \\
\hline 10 & 0,566128 & 90,722 & 9,278 \\
\hline 11 & 0,566167 & 90,718 & 9,282 \\
\hline 12 & 0,566195 & 90,718 & 9,282 \\
\hline
\end{tabular}

Fonte: Elaboração própria

Através da tabela 3 foi possível verificar que a influencia do crédito total sobre de juros é de $9,282 \%$. Normalmente, considera-se um percentual ideal de influencia acima de $10 \%$. Porém, o nível de influencia do crédito sobre os juros não é suficientemente baixo de forma a ser ignorado. Portanto, optou-se por realizar o impulso-resposta.

Figura 3 - Impulso resposta de crédito total sobre juros total 


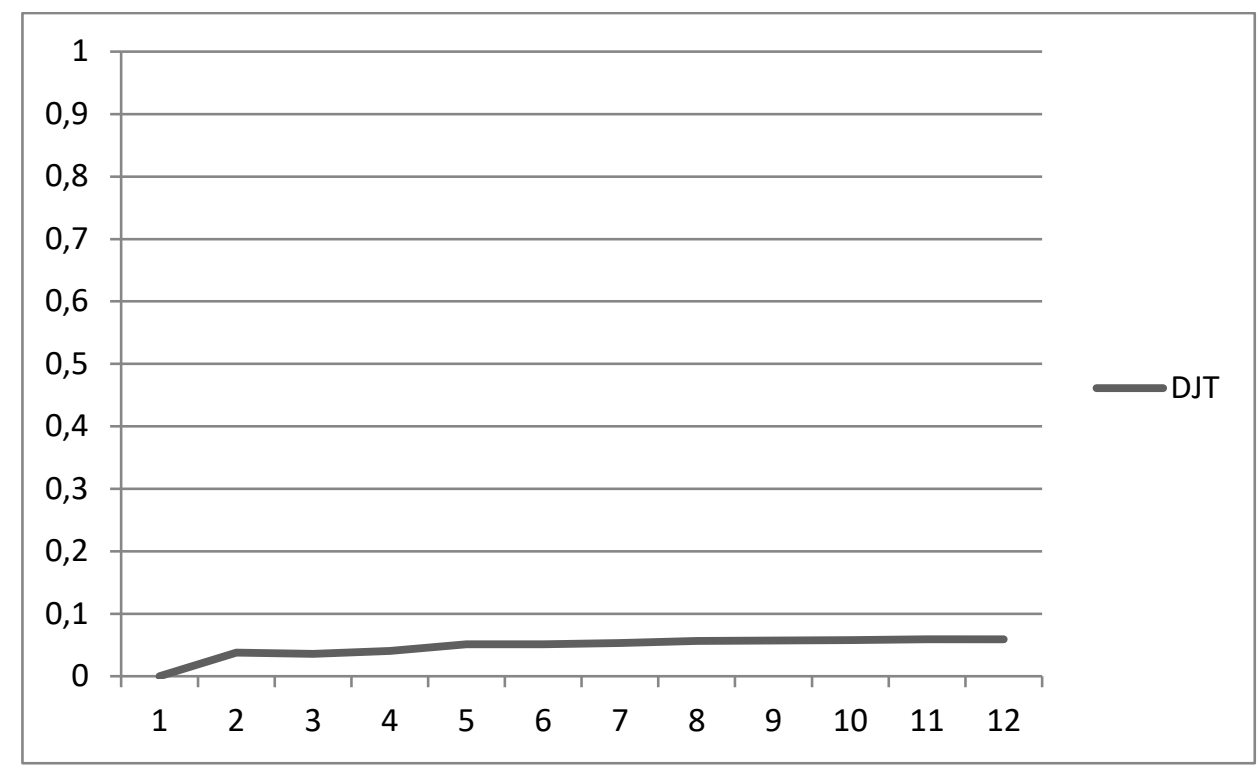

Fonte: Elaboração própria.

Nota: Impulso referente a um choque de 1\% na variável de referência.

A figura 3 indica que apesar do crédito total exercer influência sobre a taxa de juros a magnitude desta influência e baixa. Quando se observa a resposta a choques, é possível perceber que o choque de elevação de $1 \%$ nos créditos eleva os juros totais em $0,05 \%$, ou seja, a resposta dos juros a mudanças no volume de credito total contratado é tão pequena que pode ser considerada nula.

Tabela 4 - Decomposição da variância da série juros total

\begin{tabular}{l|l|l|l}
\hline Passos & Erro padrão & Juros total & Crédito total \\
\hline 1 & 7,160336 & 63,348 & 36,652 \\
\hline 2 & 8,415239 & 47,954 & 52,046 \\
\hline 3 & 9,479842 & 53,923 & 46,077 \\
\hline 4 & 9,946988 & 58,074 & 41,926 \\
\hline 5 & 10,05551 & 58,297 & 41,703 \\
\hline 6 & 10,09322 & 58,282 & 41,718 \\
\hline 7 & 10,11694 & 58,476 & 41,524 \\
\hline 8 & 10,12654 & 58,519 & 41,481 \\
\hline 9 & 10,12888 & 58,509 & 41,491 \\
\hline 10 & 10,13022 & 58,52 & 41,48 \\
\hline 11 & 10,13111 & 58,526 & 41,474 \\
\hline 12 & 10,13126 & 58,525 & 41,475
\end{tabular}

Fonte: Elaboração própria

Entretanto, a influência das taxas de juros sobre o credito total (Tabela 4) é relativamente grande, uma vez que juros são responsáveis por $58,525 \%$ da variância do crédito. Ambos os resultados de decomposição da variância estão de acordo com o observado na economia. As taxas de juros básicas são determinadas pelo COPOM, sendo assim, mudanças no nível de credito contratados realmente não devem impactar nos juros, ou seja, a taxa de juros é uma variável exógena neste caso.

Revista de Estudos Sociais | Ano 2017, N. 39, V. 20, Pag. 176 
Figura 4 - Impulso resposta de juros total sobre crédito total

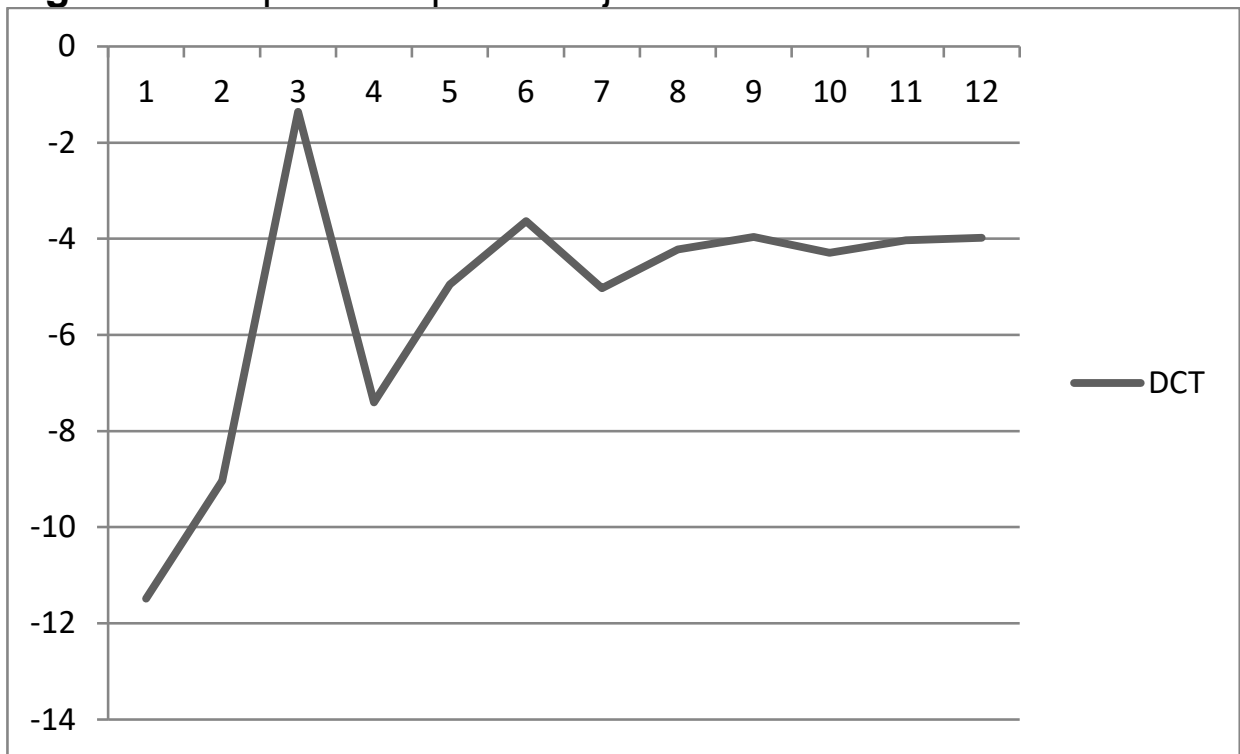

Fonte: Elaboração própria.

Nota: Impulso referente a um choque de $1 \%$ na variável de referência.

Ao observar o impulso resposta (Figura 4), é possível verificar que um aumento de $1 \%$ nas taxas de juros causa uma redução de $4 \%$ na tomada total de crédito na economia. O sinal está de acordo com o previsto na economia. Ao contrário da baixa influencia do credito sobre juros (gráfico anterior), pode-se verificar uma influencia alta dos juros nas decisões de contratação de crédito.

Cabe agora, verificar se há diferenças entre a influência dos juros sobre crédito de pessoa física e crédito de pessoa jurídica.

\section{Crédito de pessoa jurídica}

O teste de cointegração, como dito anteriormente, é utilizado para verificar se o modelo estimado será um VAR ou um VEC.

Tabela 5 - Teste de cointegração entre juros e crédito de pessoa jurídica

\begin{tabular}{l|l|l|l|l|l|l|l}
\hline $\mathrm{p}-\mathrm{r}$ & $\mathrm{r}$ & Autovalor & Traço & Traço* $^{*}$ & Perc95 & P-Valor & P-Valor \\
\hline 2 & 0 & 0.082 & 7.483 & 6.842 & 20.164 & 0.860 & 0.901 \\
\hline 1 & 1 & 0.022 & 1.547 & 1.398 & 9.142 & 0.855 & 0.879 \\
\hline
\end{tabular}

Fonte: Elaboração própria

Não foi possível verificar o vetor de cointegração para juros e crédito de pessoa jurídica, pois o $p$-valor ${ }^{*}=0,901$ indica que não é possível rejeitar a hipótese de 0 vetores de cointegração. Sendo assim, para este modelo foi estimado um VAR com variáveis em diferença.

Tabela 6 - Decomposição da variância da série juros para pessoa jurídica

\begin{tabular}{l|l|l|l}
\hline Passos & Erro padrão & Juros (PJ) & Crédito (PJ) \\
\hline 1 & 0,430 & 100,000 & 0,000 \\
\hline 2 & 0,470 & 83,602 & 16,398 \\
\hline 3 & 0,474 & 83,873 & 16,127 \\
\hline
\end{tabular}

Revista de Estudos Sociais | Ano 2017, N. 39, V. 20, Pag. 177 


\begin{tabular}{l|l|l|l}
\hline 4 & 0,488 & 84,700 & 15,300 \\
\hline 5 & 0,492 & 83,103 & 16,897 \\
\hline 6 & 0,493 & 83,101 & 16,899 \\
\hline 7 & 0,494 & 83,190 & 16,810 \\
\hline 8 & 0,495 & 83,000 & 17,000 \\
\hline 9 & 0,495 & 82,995 & 17,005 \\
\hline 10 & 0,495 & 83,004 & 16,996 \\
\hline 11 & 0,495 & 82,981 & 17,019 \\
\hline 12 & 0,495 & 82,979 & 17,021 \\
\hline
\end{tabular}

Fonte: Elaboração própria

Através da Tabela 6 verificou-se que o crédito para pessoa jurídica possui influência de $17 \%$ sobre a taxa de juros de pessoa jurídica. Portanto, o crédito empresarial consegue afetar a determinação da taxa de juros. Isso é um sinal de que o empréstimo para investimento afeta a formação dos juros e isso pode ser um indicativo da existência de política de crédito para o setor produtivo que seja capaz de fazer com que variações de crédito afetem juros.

Figura 5 - Impulso resposta de crédito sobre juros de pessoa jurídica

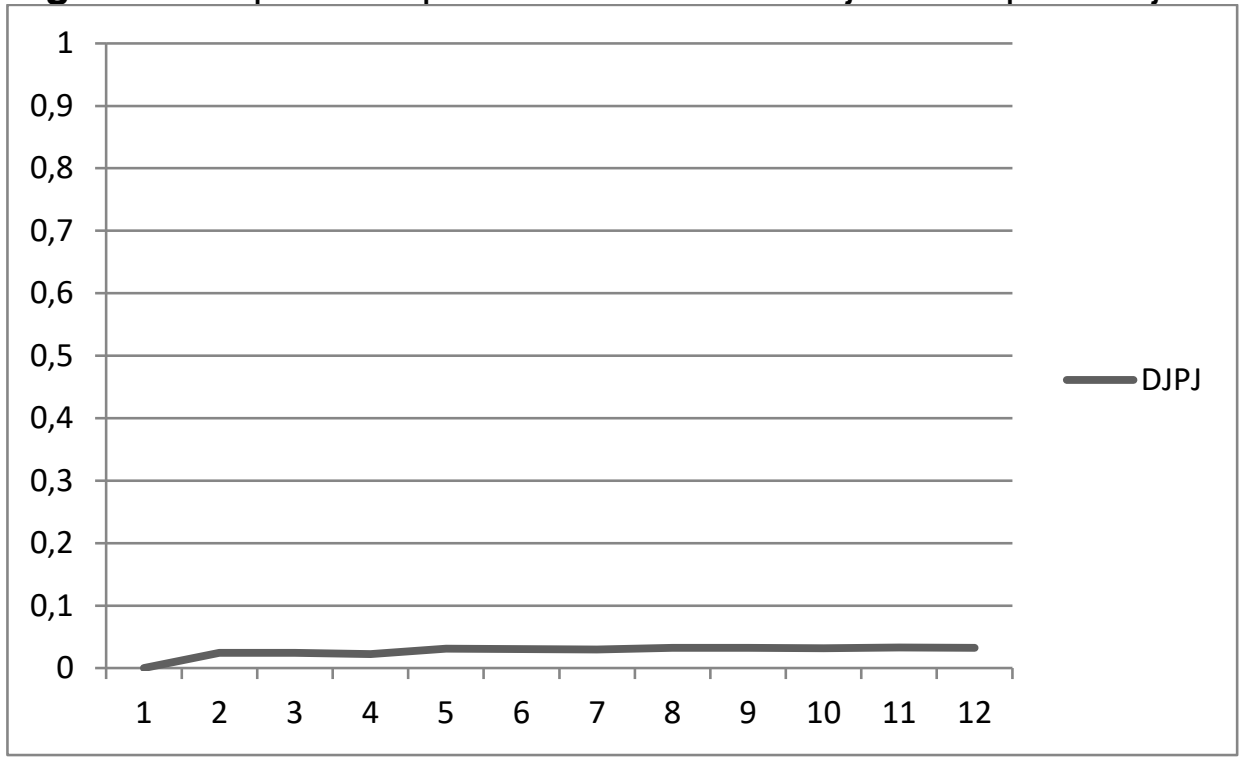

Fonte: Elaboração própria.

Nota: Impulso referente a um choque de 1\% na variável de referência.

Porém, o grau em que o aumento de crédito consegue afetar a taxa de juros é extremamente baixo. O aumento do crédito produtivo em $1 \%$ faz com que a taxa de juros aumente apenas em $0,03 \%$.

Tabela 7 - Desomposição da variância da série crédito de pessoa jurídica

\begin{tabular}{l|lll}
\hline Passos & Erro padrão & Juros $(\mathrm{PJ})$ & Crédito $(\mathrm{PJ})$ \\
\hline 1 & 10,154 & 40,634 & 59,366 \\
\hline 2 & 12,178 & 31,180 & 68,820 \\
\hline 3 & 12,510 & 34,313 & 65,687 \\
\hline
\end{tabular}

Revista de Estudos Sociais | Ano 2017, N. 39, V. 20, Pag. 178 


\begin{tabular}{l|l|l|l}
\hline 4 & 13,228 & 37,275 & 62,725 \\
\hline 5 & 13,495 & 36,600 & 63,400 \\
\hline 6 & 13,528 & 36,824 & 63,176 \\
\hline 7 & 13,619 & 37,173 & 62,827 \\
\hline 8 & 13,658 & 37,083 & 62,917 \\
\hline 9 & 13,663 & 37,107 & 62,893 \\
\hline 10 & 13,675 & 37,158 & 62,842 \\
\hline 11 & 13,681 & 37,146 & 62,854 \\
\hline
\end{tabular}

Fonte: Elaboração própria

Quando se observa a decomposição da variância da série de crédito de pessoa jurídica (Tabela 7) é possível perceber que a taxa de juros de pessoa jurídica tem influencia de $37 \%$ sobre as variações de crédito de pessoa jurídica. Este é um resultado esperado visto que os investimentos são influenciados em parte pelos juros.

Figura 6 - Impulso resposta de juros sobre crédito de pessoa jurídica

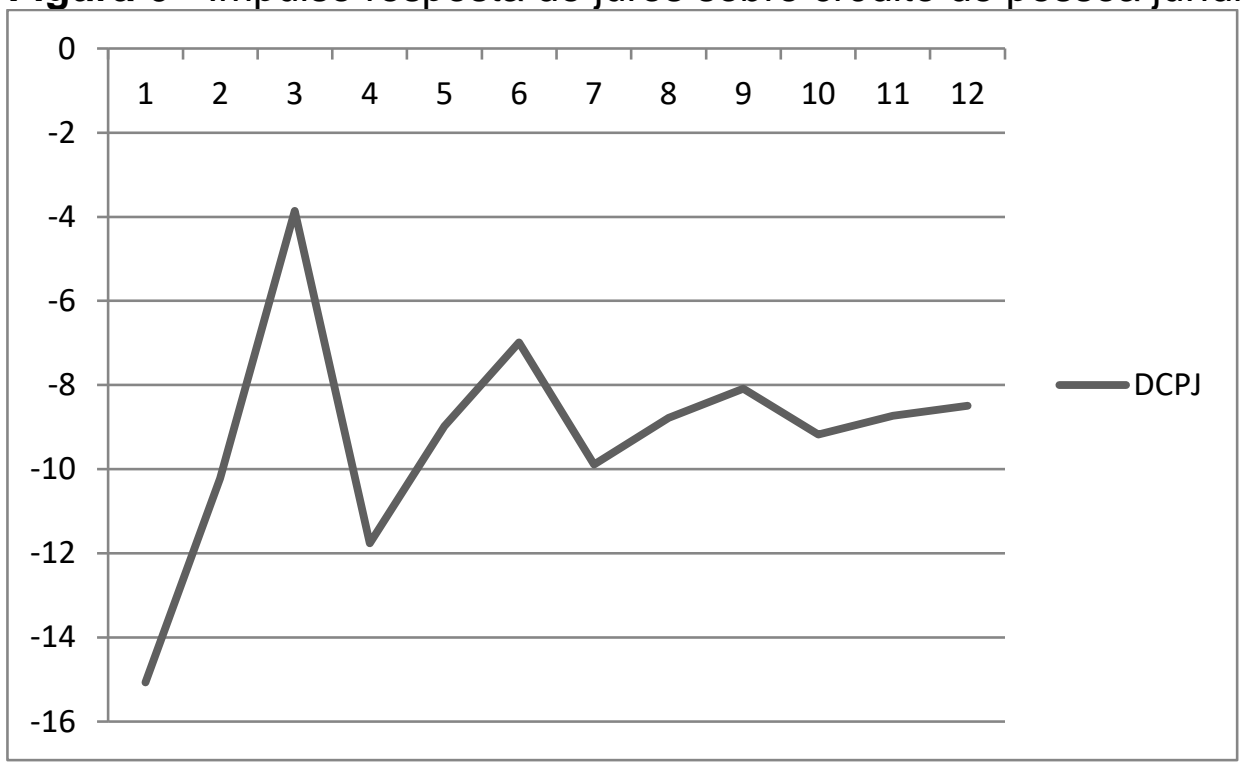

Fonte: Elaboração própria.

Nota: Impulso referente a um choque de 1\% na variável de referência.

Novamente, quando se observa o impacto da elevação de $1 \%$ nos juros PJ é possível verificar que há uma queda de $8 \%$ na tomada de crédito de PJ. Isto indica que a tomada de crédito é bastante sensível a mudanças nos juros.

\section{Crédito de pessoa física}

O primeiro passo é o teste de cointegração para verificar se será utilizado o VAR ou VEC na estimação do modelo de crédito de pessoa física.

Tabela 8 - Teste de cointegração para juros e crédito de pessoa jurídica

\begin{tabular}{l|l|l|l|l|l|l|l}
\hline p-r & r & Autovalor & Traço & Traço* $^{*}$ & Perc95 & P-Valor & P-Valor* \\
\hline 2 & 0 & 0.218 & 19.802 & 18.510 & 20.164 & 0.056 & 0.085 \\
\hline
\end{tabular}

Revista de Estudos Sociais | Ano 2017, N. 39, V. 20, Pag. 179 


\begin{tabular}{l|l|l|l|l|l|l|l}
\hline 1 & 1 & 0.041 & 2.857 & 2.276 & 9.142 & 0.615 & 0.723 \\
\hline
\end{tabular}

Fonte: Elaboração própria

O teste de cointegração indica que não há vetor de cointegração entre juros e crédito pessoa física. Sendo assim, será estimado um modelo VAR com variáveis em diferença.

Tabela 9 - Decomposição da variância da série juros de pessoa física

\begin{tabular}{l|l|l|l}
\hline Passos & Erro padrão & Juros (PF) & Crédito (PF) \\
\hline 1 & 0,669 & 100,000 & 0,000 \\
\hline 2 & 0,702 & 97,174 & 2,826 \\
\hline 3 & 0,703 & 97,095 & 2,905 \\
\hline 5 & 0,706 & 97,114 & 2,886 \\
\hline 6 & 0,706 & 97,073 & 2,927 \\
\hline 7 & 0,706 & 97,064 & 2,936 \\
\hline 8 & 0,706 & 97,064 & 2,936 \\
\hline 10 & 0,706 & 97,064 & 2,936 \\
\hline 11 & 0,706 & 97,063 & 2,937 \\
\hline 12 & 0,706 & 97,063 & 2,937 \\
\hline & 0,706 & 97,063 & 2,937 \\
\hline & 0,706 & 97,063 & 2,937
\end{tabular}

Fonte: Elaboração própria

Pode-se observar pela Tabela 9 que a tomada de crédito de pessoa física possui influencia muito baixa sobre a taxa de juros. Este resultado está de acordo com o esperado, pois a taxa de juros é um instrumento de política monetária determinada pelo COPOM e, portanto variações na tomada de crédito para consumo não afetam a formação dos juros. Como a influência do credito (pf) sobre juros (pf) é pequeno não será mostrado o impulso resposta.

Tabela 10 - Decomposição da variância da série crédito de pessoa física

\begin{tabular}{l|l|l|l}
\hline Passos & Erro padrão & Juros (PF) & Crédito (PF) \\
\hline 1 & 5,570 & 40,227 & 59,773 \\
\hline 2 & 6,355 & 32,424 & 67,576 \\
\hline 3 & 6,777 & 38,601 & 61,399 \\
\hline 4 & 6,864 & 39,858 & 60,142 \\
\hline 5 & 6,889 & 39,737 & 60,263 \\
\hline 6 & 6,896 & 39,747 & 60,253 \\
\hline 7 & 6,899 & 39,798 & 60,202 \\
\hline 9 & 6,900 & 39,802 & 60,198 \\
\hline 10 & 6,900 & 39,800 & 60,200 \\
\hline 11 & 6,900 & 39,801 & 60,199 \\
\hline 12 & 6,900 & 39,801 & 60,199 \\
\hline
\end{tabular}

Fonte: Elaboração própria

Revista de Estudos Sociais | Ano 2017, N. 39, V. 20, Pag. 180 
Porém, quando se observa a decomposição da variância do crédito, verifica-se que as taxas de juros (pf) tem influência sobre a contratação de crédito (pf) de quase $40 \%$.

Figura 7 - Impulso resposta de juros total sobre crédito de pessoa física

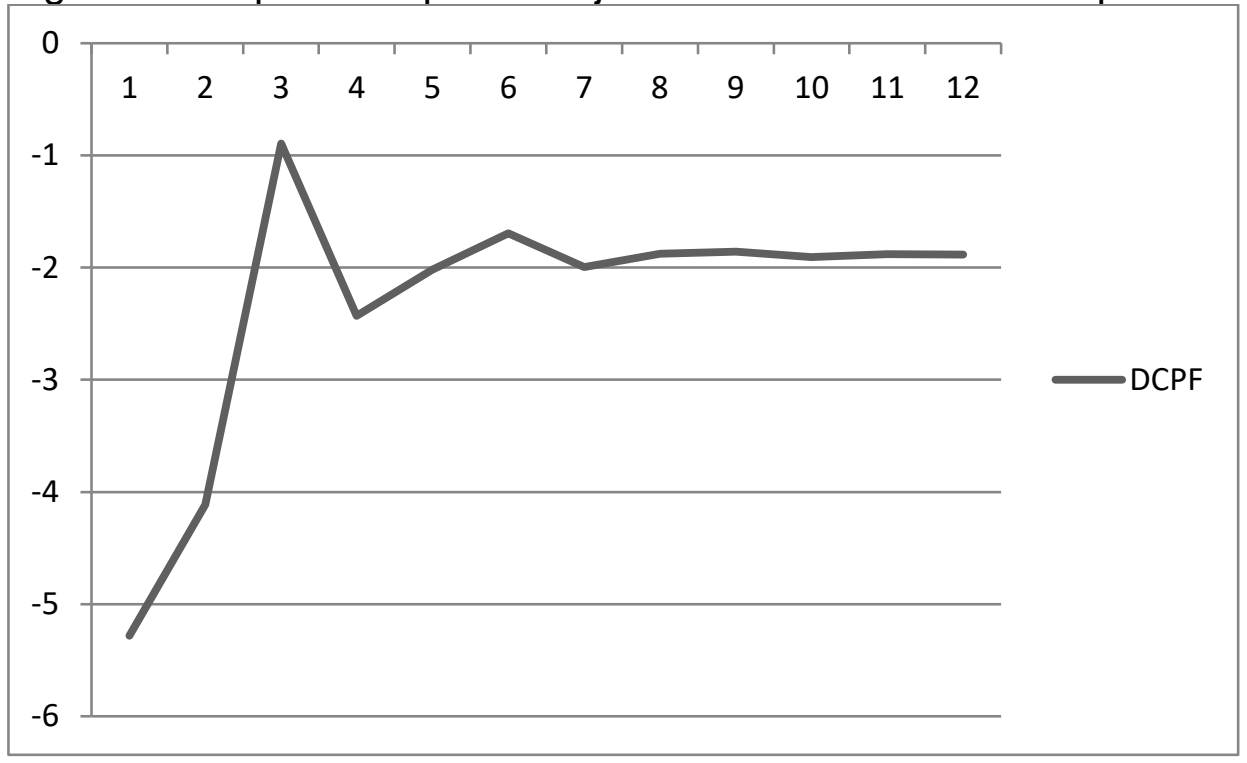

Fonte: Elaboração própria.

Nota: Impulso referente a um choque de $1 \%$ na variável de referência.

Pelo impulso resposta (Figura 5) é possível verificar que elevações na taxa de juros de $1 \%$ causa a redução na tomada de crédito para pessoa física de $2 \%$. O resultado da influência dos juros sobre o crédito demonstra que o crédito reage a juros, porém os juros reagem pouco a crédito. Ademais, é possível verificar que a reação no setor produtivo é maior que para pessoa física.

\section{Considerações finais}

$\mathrm{O}$ trabalho teve como objetivo verificar o relacionamento entre juros e crédito. De modo geral, verificou-se que a influencia dos juros sobre crédito é bastante superior à influência do crédito sobre juros. Este era um resultado esperado visto que os juros são utilizados como instrumento de política monetária e, portanto, possui alta exogenia. Quando se analisam produtores e consumidores há resultados discrepantes. Um deles é de que os produtores (pessoa jurídica) possuem algum grau de influência sobre a formação das taxas de juros enquanto que a influência dos consumidores (pessoa física) sobre juros é praticamente inexistente. Este resultado pode indicar que as políticas de fomento à produção adotadas pelo governo fazem com que os tomadores de crédito para investimento possuam algum grau de manobra na determinação dos juros. O outro resultado é de que os consumidores reagem menos a aumento de juros do que produtores. Isto pode ser pelo fato de o produtor visar lucro enquanto que o consumidor visa a satisfação. Sendo assim, uma possibilidade é de que os consumidores realizem a tomada de crédito por impulso fazendo com que a redução na contratação de crédito dada uma alta na taxa de juros seja menos sentida para consumidores que para produtores.

Revista de Estudos Sociais | Ano 2017, N. 39, V. 20, Pag. 181 
Referências Bibliográficas

BACEN. Nota para a Imprensa de Política Monetária e Operações de Crédito Nova estrutura de dados de crédito - Nota Metodológica, Banco Central do Brasil,201-, Disponivel em: https://www.bcb.gov.br/ftp/infecon/notaempr.pdf Acesso em julho de 2017.

BISCHOFF, L. Risco bancário e taxa de juros: estudo empírico em instituições financeiras no Brasil. UNB. Dissertação. 2015

CARVALHO, F C et al. Economia Monetária e Financeira: Teoria e Política. 2ed. Rio de Janeiro: Campus, 2007.

CORAZZA, G. Crise e Reestruturação Bancária no Brasil. 2000. Disponível em: www.ppge.ufrgs.br/ppge/pcientifica/2000 08.pdf Acesso em abril de 2017.

CUTRUNEO, T. B. Regras, Estabilização e Monetarismo. Fundação Getúlio Vargas. $\quad$ Dissertação. 2010.2 Disponível em http://bibliotecadigital.fgv.br/dspace/bitstream/handle/10438/5906/63080100018 .pdf? sequence $=1$ Acesso em dezembro de 2016.

CYSNE, R P; COSTA, S G S. Reflexos do Plano Real sobre o sistema bancario brasileiro. Revista Brasileira de Economia. Rio de Janeiro jul/set 1997.

DICKEY, D.A. ; FULLER, W.A. Distribution of the estimators for autoregressive time series with a unit root. Journal of the American Statistical Association, Alexandria, 74, p. 427-431, 1979.

DICKEY, D.A. ; FULLER, W.A. Likelihood ratio statistics for autoregressive time series with a unit root. Journal of the American Statistical Association, Alexandria, 49, p. 1057-1072, 1981

ENDERS, W. Applied econometric time series. New York:Wiley, 2004

FERREIRA, M.A.M. et al. Previsão de risco de crédito para a definição do perfil de clientes de um banco de varejo. Revista de negócios, Blumenau, v.16, n.2, p. 47- 64, 2011.

FRIEDMAN, M. A program for monetary stability. Fordham University Press, 1960

FULLER, W.A. Introduction to statistical time series. New York. John Wiley \& Sons, 1976

GONÇALVES, JR et al. Inadimplência no crédito agrícola: uma aplicação do modelo de regressão logística. Sociedade Brasileira de Economia, Administração e Sociologia Rural. Rio Branco, 20 a 23 de julho de 2008 ISHII, K S. A Teoria de Área Monetária Ótima para o Brasil: uma análise das diferenças regionais. Tese, USP. 2008

JOHANSEN, S. Statistical analysis of cointegration vectors. Journal of Economic Dynamics and Control, Boston, n. 12, p. 231-254, 1988.

JUNQUEIRA, R G P; ABRAMOVAY, R. A Sustentabilidade das Microfinanças Solidárias. Revista de Administração, São Paulo, v.40, n.1, p.19-33, jan./fev./mar. 2005

LUCAS, R. Expectations and the neutrality of money. Journal of Economic Theory, v.4, n.2, p.103-124, apr, 1972

MISHKIN, F. Moedas, bancos e mercados financeiros. Rio de Janeiro: LTC, 1998.

MONTICELLI, R. Taxas de juros, crédito e inadimplência. Monografia. Porto Alegre, 2003.

Revista de Estudos Sociais | Ano 2017, N. 39, V. 20, Pag. 182 
NASCIMENTO, C.E.L. A evolução recente do crédito e sua participação no combate a crise financeira internacional. Jornal eletrônico Faculdades Integradas Vianna Junior. 2015.

PEREIRA, A.P; WAGNER,E.M.. Racionamento de crédito no brasil: um estudo de causalidade. Encontro de Economia Catarinense. 2012.

SANT'ANNA, A A; BORÇA JUNIOR, G R; ARAUJO, PEDRO Q. Mercado de Crédito no Brasil: Evolução Recente e o Papel do BNDES (2004-2008) Revista BNDES, Rio De Janeiro, V. 16, N. 31, P. 41-60, JUN. 2009

SBICCA, A; FLORIANI, V; JUK,Y. Expansão do crédito no Brasil e a vulnerabilidade do consumidor. Revista Economia \& Tecnologia (RET). Volume 8, Número 4, p. 05-16, Out/Dez 2012

SIMS, C.A. Macroeconomic and reality. The American Economic Review, Pittsburgh, v.48, n. 1, p. 1-49, Jan. 1980.

TABAK, B M; CRAVEIRO, G L.; CAJUEIRO, D O. Eficiência Bancária e Inadimplência: testes de Causalidade. Trabalhos para Discussão Brasília $n^{\circ}$ 220 out. 2010 p. $1-36$

TAVARES, D P; MONTES, G C; GUILLÉN, O T C. Transmissão da política monetária pelos canais de tomada de risco e de crédito: uma análise considerando os seguros contratados pelos bancos e o spread de crédito no Brasil. Trabalhos para Discussão Brasília ํㅡ은 308 julho 2013 p. 1-23

TOMAZZIA, E. C.; MEURER, R. O mecanismo de transmissão da política monetária no Brasil: uma análise em VAR por setor industrial. Revista de Economia Aplicada, v. 13, p. 371-398, 2009.

WOOFORD, M. Inflation Targeting and Optimal Monetary Policy. Princeton University. Federal Reserve Bank of St. Louis, October 16-17, 2003. 